\title{
Neurofeedback for the treatment of children and adolescents with ADHD: a randomized and controlled clinical trial using parental reports
}

\author{
Nezla S Duric ${ }^{1,2^{*}}$, Jørg Assmus ${ }^{3+}$, Doris Gundersen ${ }^{4+}$ and Irene B Elgen ${ }^{5,6}$
}

\begin{abstract}
Background: A randomized and controlled clinical study was performed to evaluate the use of neurofeedback (NF) to treat attention-deficit/hyperactivity disorder (ADHD) in children and adolescents.

Methods: The ADHD population was selected from an outpatient clinic for Child and Adolescent Mental Health in Norway. Ninety-one of the 275 children and adolescents ranging in age from 6 to 18 years (10.5 years) participated in 30 sessions of an intensive NF program. The reinforcement contingency was based on the subjects' production of cortical beta 1 activity $(15-18 \mathrm{~Hz})$. The ADHD participants were randomized into three groups, with 30 in the NF group, 31 controls in a group that was given methylphenidate, and 30 in a group that received NF and methylphenidate. ADHD core symptoms were reported by parents using the parent form of the Clinician's Manual for Assessment by Russell A. Barkley.
\end{abstract}

Results: Ninety-one children and adolescents were effectively randomized by age, sex, intelligence and distribution of ADHD core symptoms. The parents reported significant effects of the treatments, but no significant differences between the treatment groups were observed.

Conclusions: NF was as effective as methylphenidate at treating the attentional and hyperactivity symptoms of ADHD, based on parental reports.

Trial registration: Current Controlled Trials NCT01252446

Keywords: Attention-deficit/hyperactivity disorder (ADHD), Neurofeedback, Barkley rating scale for parents

\section{Background}

Attention-deficit/hyperactivity disorder (ADHD) is a developmental mental disorder characterized by persistent symptoms of inattention or inattention in combination with hyperactivity and impulsivity. Its prevalence may be up to one in four $[1,2]$. The severity of these symptoms and its enduring nature are known to impair a person's capacity to effectively function. Treatment programs using behavioral and pharmacotherapeutic approaches are well established [3,4]. The Multimodal Treatment study (MTA) of ADHD identified advantages of

\footnotetext{
*Correspondence: nezlad@gmail.com

${ }^{\dagger}$ Equal contributors

'Department of Child and Adolescent Psychiatry, Helse Fonna Haugesund

Hospital, Vinjesgate 10, Haugesund 5501, Norway

${ }^{2}$ Center for Child and Adolescent Mental Health, University of Bergen,

Bergen, Norway

Full list of author information is available at the end of the article
}

multimodal treatment $[1,5,6]$. Because of rapid improvements in attention and reduced hyperactivity, many children with ADHD have been treated with stimulants [79]. However, improvements in social and academic skills following stimulant treatment have not been reported [9]. In addition, concerns with the benefits and side effects of long-term stimulant treatment have also been noted $[10,11]$.

Many clinical trials have found that neurofeedback (NF) effectively treats the symptoms of ADHD $[12,13]$. The effects of NF have been described as improved attention, decreased hyperactivity, and increased academic and social skills $[1,14,15]$. However, other studies have only found improvements in attention [16], and two studies with large sample sizes did not find significant improvements in core ADHD symptoms $[11,17]$.

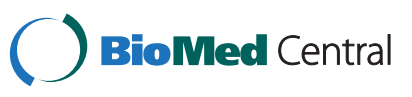

(c) 2012 Duric et al.; licensee BioMed Central Ltd. This is an Open Access article distributed under the terms of the Creative Commons Attribution License (http://creativecommons.org/licenses/by/2.0), which permits unrestricted use, distribution, and reproduction in any medium, provided the original work is properly cited. 
NF has been discussed as being an effective ADHD treatment when given alone or in combination with medications [11]. One study did not find significant treatment responses between Ritalin and NF [17].

$\mathrm{NF}$ is a learning process in which the brain is rewarded for positive changes in its activity [13]. The response to this learning process is visual or auditory feedback. In NF, the placement of the electrodes and the frequency of stimulation are important. The International 10-20 system is a recognized method to describe and apply the location of scalp electrodes in the context of an electroencephalography (EEG) exam or experiment. Most NF studies have used the standardized electrode placements $\mathrm{Cz}, \mathrm{C} 3$, and C4 [14]. Some studies included frontal electrodes when using NF to treat ADHD, such as the Fz, F3, and F4 electrodes [14]. Several NF protocols for treating ADHD are available. Single-channel protocols (unipolar) developed by Lubar and interhemispheric (bipolar) protocols developed by Othmers are widely practiced and supported by largescale clinical studies [18-20].

Different approaches regarding NF treatment have been developed for ADHD [1,12,21]. The most frequently used frequencies enhance beta $(15-18 \mathrm{~Hz})$ and inhibit theta $(4-7 \mathrm{~Hz})$ brain activity [22]. Sensorimotor rhythm (SMR; 12-15 Hz) protocols that enhance SMR activity (low beta activity), alpha/theta $(8-11 \mathrm{~Hz} / 4-$ $7 \mathrm{~Hz}$ ) protocols that enhance alpha brain activity, and SCP (slow cortical potentials) protocols that reward polarity changes in EEGs are also used [23]. Usually no more than two different treatment protocols are used in NF treatments [22].

The lack of large and controlled studies may have limited the use of NF treatments in the clinic [24-27]. Many prospective controlled studies have used either stimulants or waiting lists as the control groups [17]. Only a few studies were randomized and controlled trials [16,17,28-30].

In addition, several non-randomized studies found a large effect size (ES) for attention and a medium ES for hyperactivity [31], but a randomized study by Arns and Linden found a small ES for hyperactivity [14,32].

Use of sham feedback (placebo) for evaluating the efficacy of NF in the ADHD population was declared unethical by the University of California, San Diego $[24,33]$. This is the most likely reason why standard medication treatments have been applied to the control groups in NF research studies.

The present study was a controlled and randomized clinical study that included children and adolescents with ADHD, who were followed at a Child and Adolescent Mental Health Clinic in Norway. The aim of the study was to evaluate the effects of NF on the core symptoms of ADHD, including attention and hyperactivity.

\section{Methods}

\section{Subjects}

Over a period of 3 years (2007-9), 628 children and adolescents aged six through 18 years were referred for the treatment of ADHD to the Child and Adolescent Mental Health Clinic, Haugesund Hospital, Rogaland County in Norway. Of these, 285 (45\%) met the criteria for ADHD according to the International Classification of Disease (ICD-10) (Figure 1). All children and adolescents with ADHD were invited to participate in the study. Ten of these subjects were excluded because of a low IQ $(<70)$, and 155 refused to participate. These children were of a similar gender and age as the participants. After randomization, 39 children and adolescent dropped out of the study: 13 randomized to NF group, 15 to medication group and 11 belonged to combined NF/medication group. The treatment was completed successfully in 91 (70 \%) children and adolescents (Figure 1).

\section{ADHD}

The ADHD-referred population through 1 year (2007) has been described in detail in earlier papers [34,35]. Children referred for ADHD underwent a clinical assessment. The assessment included a child appropriate medical examination, a clinical psychiatric interview, and observations to assess ADHD and other appropriate diagnoses. Questionnaires regarding ADHD were filled in by the child, or by the parent or teacher of the child. The medical examination was done to exclude somatic conditions as the cause of the ADHD symptoms. A child psychiatrist evaluated the assessments and categorized the child as having ADHD or a nonADHD condition according to ICD-10 diagnostic criteria.

\section{Inclusion criteria}

All children with ADHD met the following criteria to be included in the study: 1) symptomatology consistent with ICD10 criteria for the diagnosis of ADHD; 2) age 6-18 years; 3 ) cognitive function above IQ 70. The children were evaluated using the Wechsler Intelligence Scale for Children (WISC-IV) [36,37].

\section{Treatment groups}

The children with ADHD were randomly placed into three groups: 1) the NF group received NF; 2) the NF + MED group received NF and methylphenidate (MPH); and 3) the MED group received MPH (Figure 1).

\section{NF}

NF is designed to change certain types of EEG activity $[38,39]$. The goal of the NF treatment was to enhance beta and depress theta activity [19]. 


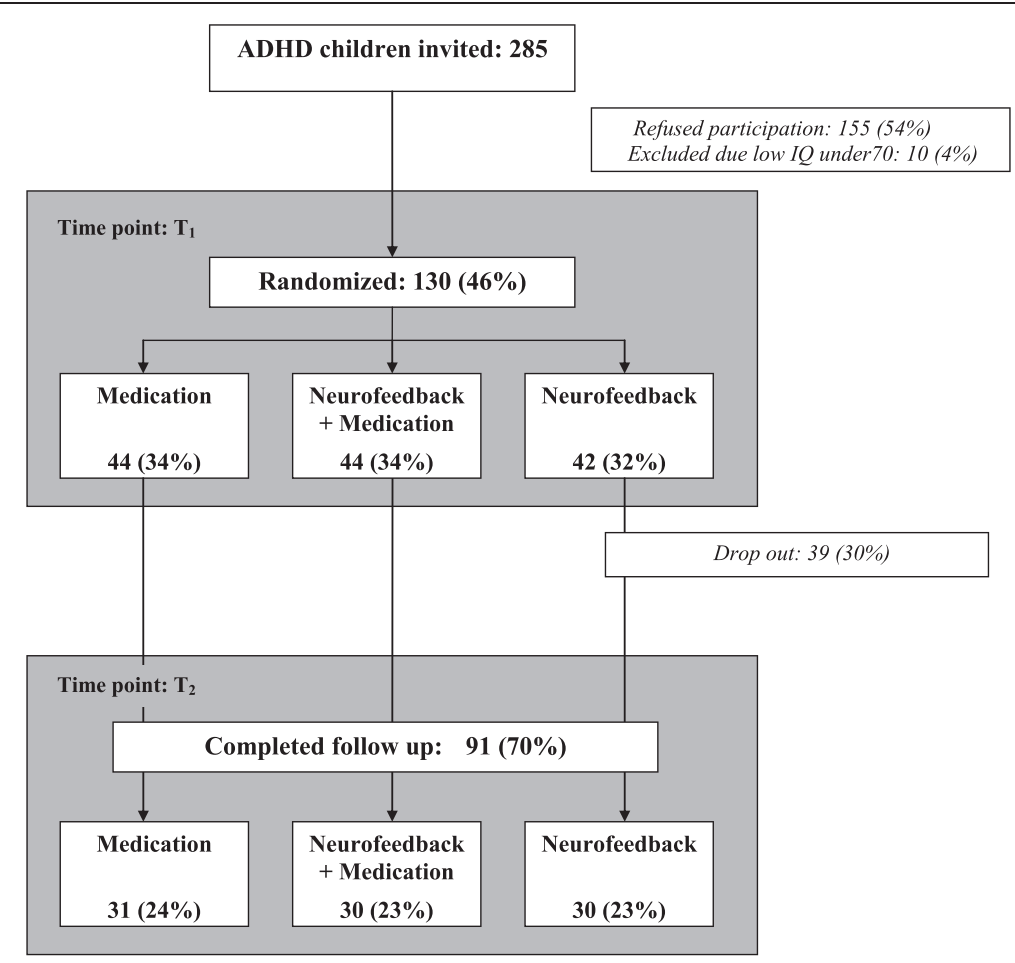

Figure 1 Clinical population of children with ADHD who were invited to participate in this randomized neurofeedback trial.

During the NF treatment, the patient received feedback regarding their own brain activity. Unipolar placed sensors on the scalp measured brain activity, and a computer processed the signals as brainwave frequencies. The flow of this activity is shown to the subject, who attempts to change the activity level. Some frequencies are susceptible to promotion and others are susceptible to being diminished. Such information was presented to the children and adolescents in the form of a video game or film. When the children and adolescents played the video game or watched the films, they produced brainwave activity that was "shaped" toward more regulated performance. NF in the study was conducted using the Infinity software and equipment supplied by the biofeedback/NF and psycho-physiological instrument manufacturer Thought Technology [40].

A beta/theta NF treatment protocol for ADHD was used, including the appropriate frequency ranges for the EEGs and electrode placements. Beta enhancement (16-20 HZ) and Theta suppression (4-7 Hz) were assessed. The treatment site used was in the central area and unipolar electrodes was placed on the $\mathrm{Cz}$, whereas the ground electrode was placed on the earlobe [18].

NF was conducted three times a week with 30 treatments for each child and adolescent. Each treatment lasted 40 minutes and was separated into pre and post 5-minute baseline periods (alpha training) and 30 minutes of NF (beta/theta training). Theta activity was defined as $4-7 \mathrm{~Hz}$, alpha activity as $8-12 \mathrm{~Hz}$, SMR activity as $13-15 \mathrm{~Hz}$, beta activity as $16-20 \mathrm{~Hz}$, and electromyography (EMG) activity as $80-150 \mathrm{~Hz}$ activity [41]. The events above these threshold levels were monitored. The standards used in the treatment were to decrease theta activity by inhibiting high amplitude theta activity or by rewarding high amplitude beta activity. The treatment effect was defined as increased beta/SMR activity of $13-20 \mathrm{~Hz}$, decreased theta activity of $4-7 \mathrm{~Hz}$, and decreased EMG activity.

Subjects in the MED and NF/MED group were administered methylphenidate (MPH) twice per day at the recommended dose $1 \mathrm{mg}$ per $\mathrm{kg}$ with the final medication doses from 20 to $60 \mathrm{mg}$ daily doses.

\section{Pre- and post-evaluation}

Two core symptoms of ADHD were evaluated. Attention and hyperactivity were evaluated using the Clinician's Manual for the Assessment of Disruptive Behavior Disorders - Rating Scale for Parents, from Russell A. Barkley [42]. The manual offers an effective tool for assessing attention and hyperactivity. The scale is divided into subscales for inattention and hyperactivity, and a total score. Normative data are rated according to the age (5-18 years) and gender of the subject. The children were evaluated in two different periods during the study $\left(\mathrm{T}_{1}, \mathrm{~T}_{2}\right)$. The baseline evaluation $T_{1}$ was completed prior to the treatment. 
The effects of the treatments were evaluated as a change in ADHD core symptoms from $T_{1}$ to $T_{2}$. All children underwent post-treatment evaluations approximately 1 week after the NF had been completed $\left(\mathrm{T}_{2}\right)$.

The Regional Ethics Committee on Medical Research approved the project protocol used in this study, and written consent was obtained from all subjects and their parents.

\section{Statistical Analysis}

Basic descriptive methods (descriptive, Analyses of Variance (ANOVA), exact $\chi^{2}$ test) were used to investigate the baseline data. The treatment effects were investigated using a general linear model (GLM) for repeated measures, which was implemented for each of the subscales (total score, inattention, and hyperactivity). The model included the raw scores at both time points as dependent variables and the treatment groups as factors. We fitted both an unadjusted model and a model adjusted for sex and age. Even if the adjusted model may be more complete and more reliable, it was reasonable to examine the unadjusted model because of the small sample size and concomitant lack of power in the adjusted model. In the GLM, we estimated both withinsubjects contrasts to test treatment-induced changes in the ratings and between-groups effects to test for differences between the treatments. Significant differences were further examined using post-hoc tests. Additionally, we estimated the standardized ES $\delta_{\mathrm{RM}}$ for each treatment change [43].

Note that we used a negative definition of the treatment changes $\left(T_{1}-T_{2}\right)$ because we wanted an improvement associated with a positive number.

The general significance level was set to 0.05 . For the baseline investigation we had to take into account the effects of multiple comparisons. Because of the high correlation between a number of the investigated variables (Barclay ratings), a Bonferroni corrections method would be too conservative. As such, we decided to set the significance level to 0.01 , as a compromise between a Bonferroni correction and not accounting for multiple comparisons. In the GLM, we investigated only highly correlated variables, thereby reducing the number of comparisons. Therefore, we did not adjust the significance level. All computations were done using PASW 18.

\section{Results}

\section{Subjects and randomization}

There were no significant differences in demographic factors or ADHD core symptoms between the treatment groups at baseline (T1) (Table 1).

Almost one third of the subjects dropped out of the study (Figure 1). Most of them did not start the treatment because of their parents' decision (30/39) or because of the subject's decision (6). Three children did not complete the follow-up (T2) questionnaires after the NF. The 91 subjects who completed the treatment were similar to the 39 who dropped out of the treatment regarding their socio-demographic status, in terms of their family structure, number of siblings, parents' education, economic resources, and other support. There were no significant differences in their academic skills (IQ mean difference: $-9.8,95 \%$ CI: $(-7.9,5.9))$.

\section{Results within the treatment groups}

Parents reported significant changes in all scales within the three treatment groups (unadjusted, $\mathrm{p}<0.001$, Table 2). The change was quite strong for hyperactivity, but weak for attention. Consequently, the size of the change for the total scale was dominated by the hyperactivity change. After adjusting for age and sex, no significant changes were found (adjusted, Table 2).

Table 1 A clinical randomized controlled Neurofeedback study with 91 participants

\begin{tabular}{|c|c|c|c|c|c|}
\hline & $\begin{array}{c}\text { Total } \\
\text { Mean (SD) }\end{array}$ & $\begin{array}{r}\text { Medication } \\
\text { Mean (SD) }\end{array}$ & $\begin{array}{l}\text { Neurofeedback + } \\
\text { Medication } \\
\text { Mean (SD) }\end{array}$ & $\begin{array}{r}\text { Neurofeedback } \\
\text { Mean (SD) }\end{array}$ & $\mathrm{p}$-value \\
\hline \multicolumn{6}{|l|}{ Demografics } \\
\hline Children participating ${ }^{1}$ & 91 (100\%) & $31(34 \%)$ & $30(33 \%)$ & $30(33 \%)$ & \\
\hline Sex (boys) ${ }^{1}$ & $72(80 \%)$ & $27(87 \%)$ & $23(77 \%)$ & $22(73 \%)$ & $0.51^{4}$ \\
\hline Age & $11.2(2.8)$ & $10.9(2.4)$ & $11.2(2.8)$ & $11.4(3.1)$ & $0.77^{2}$ \\
\hline IQ & $87(14)$ & $87(15)$ & $85(13)$ & $89(14)$ & $0.75^{2}$ \\
\hline \multicolumn{6}{|l|}{ ADHD core symptoms ${ }^{3}$} \\
\hline Total & $34.1(8.9)$ & $35.8(10.8)$ & $33.8(7.8)$ & $32.6(7.7)$ & $0.39^{2}$ \\
\hline Attention & $16.7(4.7)$ & $17.4(5.1)$ & $17.2(4.9)$ & $15.5(4.0)$ & $0.22^{2}$ \\
\hline Hyperactivity & $17.3(5.2)$ & $18.2(6.2)$ & $16.5(4.9)$ & $17.1(4.5)$ & $0.47^{2}$ \\
\hline
\end{tabular}

${ }^{1}$ Number (\%). ${ }^{2}$ ANOVA for the null-hypotheses that the values for the randomized groups are equal. ${ }^{3}$ Barkley rating scale based on parents score.

${ }^{4}$ Exact $\mathrm{X}^{2-}$-test. 
Table 2 Parents report of ADHD core symptoms (attention, hyperactivity, total score) regarding the treatments (NF, MED, combined)

\begin{tabular}{|c|c|c|c|c|c|c|c|c|c|c|c|c|c|c|}
\hline \multirow[b]{2}{*}{ Barkley rating scores } & \multicolumn{3}{|c|}{ Pre treatment } & \multicolumn{3}{|c|}{ Post treatment } & \multirow[b]{2}{*}{$\mathrm{N}$} & \multicolumn{3}{|c|}{ Pre-Post change $\left(\mathrm{T}_{1}-\mathrm{T}_{2}\right)^{1}$} & \multirow[b]{2}{*}{ p-unadj. ${ }^{5}$} & \multirow[b]{2}{*}{ p-adj. ${ }^{6}$} & \multicolumn{2}{|c|}{ Treatment effect ${ }^{2}$} \\
\hline & $\mathrm{N}$ & Mean & $95 \% \mathrm{Cl}$ & $\mathbf{N}$ & Mean & $95 \% \mathrm{Cl}$ & & Mean $^{3}$ & $95 \% \mathrm{Cl}$ & Effect size ${ }^{4}$ & & & & p-adj. ${ }^{6}$ \\
\hline Total & & & & & & & & & & & $<.001$ & 0,310 & 0.173 & 0,228 \\
\hline Medication & 29 & 34,5 & $(30.6,38.5)$ & 22 & 27,8 & $(24.0,31.6)$ & 22 & 7.9 & $(4.5,11.4)$ & 1.79 & & & & \\
\hline Neurofeedback + Medication & 24 & 32,6 & $(28.6,36.6)$ & 22 & 23,7 & $(19.8,27.6)$ & 22 & 8,6 & $(5.0,12.2)$ & 1.76 & & & & \\
\hline Neurofeedback & 23 & 37 & $(33.9,40.0)$ & 19 & 26,7 & $(23.4,30.1)$ & 19 & 10,7 & $(7.6,13.8)$ & 2.25 & & & & \\
\hline Attention & & & & & & & & & & & $<.001$ & 0,738 & 0,098 & 0,139 \\
\hline Medication & 29 & 15,9 & $(13.8,18.0)$ & 22 & 15,2 & $(13.2,17.2)$ & 22 & 1,5 & $(-0.3,3.3)$ & 0.70 & & & & \\
\hline Neurofeedback + Medication & 24 & 15,9 & $(13.9,18.0)$ & 22 & 14,5 & $(12.0,17.2)$ & 22 & 1,1 & $(-0.7,3.0)$ & 0.46 & & & & \\
\hline Neurofeedback & 23 & 19,2 & $(17.2,21.1)$ & 19 & 16,6 & $(14.5,18.7)$ & 19 & 3,1 & $(1.6,4.5)$ & 2.08 & & & & \\
\hline Hyperactivity & & & & & & & & & & & $<.001$ & 0,077 & 0,101 & 0,186 \\
\hline Medication & 29 & 18,7 & $(16.3,21.0)$ & 22 & 12,5 & $(10.2,14.4)$ & 22 & 6,5 & $(4.3,8.6)$ & 2.45 & & & & \\
\hline Neurofeedback + Medication & 24 & 16,7 & $(14.3,19.0)$ & 22 & 9,1 & $(6.8,11.4)$ & 22 & 7,5 & $(4.9,10.0)$ & 1,75 & & & & \\
\hline Neurofeedback & 23 & 17,8 & $(15.9,19.6)$ & 19 & 10,1 & $(8.0,12.2)$ & 19 & 7,6 & $(5.6,9.6)$ & 2.88 & & & & \\
\hline
\end{tabular}

${ }^{1}$ Tests for within-subject-contrast in the General linear modell (GLM). ${ }^{2}$ Between-groups-effect in the GLM. ${ }^{3}$ Mean of the differences, i.e. excluding drop outs at one missing point. ${ }^{4}$ Effect size measure $\delta_{\mathrm{RM}}$ (ref Morris).

${ }^{5}$ Unadjusted model. ${ }^{6}$ Model adjusted for age and sex. 


\section{Results between the treatment groups}

Based on the parents' reports, we did not observe significant differences between the treatment groups. Neither the between-groups effects in the GLM nor the post-hoc tests showed any significant change scores, for any scales or pair of treatment groups (Table 2). We did not report the ESs or the results of the post-hoc tests since there were no significant differences.

Generally, our comparisons of the treatments had low powers. Although not significant, the NF group showed more than double the pre-post change in attention compared with the other two treatments (3.1 vs. 1.1 and 1.5 for the means). Additionally, we noted that the MED and NF + MED group had a confidence interval that included 0 . The NF group had the largest ES $\delta_{\mathrm{RM}}$ for all scales, but one should be careful interpreting this observation since those three scales are highly correlated. For all scales, we noted that the p-value for the unadjusted models were lower than for the adjusted models.

\section{Discussion}

In the present controlled and randomized clinical trial, NF treatment seemed to improve the core symptoms of ADHD, as assessed by parental reports. In addition, NF and MPH produced similar improvements.

The strength of the present study was the effective randomization by age, sex, and intelligence, and the distribution of ADHD core symptoms (hyperactivity and attention). However, the number of subjects in each treatment group was somewhat low.

NF improved attention and hyperactivity symptoms in children and adolescents with ADHD. This is in accordance to findings in other studies, including a study by Kaiser, Thomsen and Othmer that found significant improvements in ADHD symptoms with NF for more than three in four ADHD subjects [32,44]. We did not find significant difference between the three investigated treatments in the improvement of ADHD core symptoms. This is in accordance with the work of Rossiter and Fuchs, who found in a rather large sample size that effects of NF on hyperactivity and attention ( ES 1.011.71) are equivalent to those obtained with stimulant drugs $(0.80-1.80)[17,30]$. Consequently, NF can be suggested to produce equivalent beneficial effects for ADHD as medications. Furthermore, our findings support the suggestion by Fuchs and Lubar to introduce NF as a treatment option for children with ADHD whose parents favored a non-pharmacological treatment $[21,30]$.

However, regarding the improvement of the core symptoms of ADHD, nonspecific factors may contribute to the positive effects induced by NF $[14,16]$. Mainly, there are three nonspecific factors described in previous studies that may result in ADHD symptom improvement. These include the extraordinary amount of time spent with the therapist during NF, better motivation for changes in ADHD symptoms, and cognitivebehavioral training introduced under NF [26,32]. These factors may explain some improvement of hyperactivity, but may be a minor factor.

Even if we did not find significant differences in the core symptoms of ADHD, we observed a lower ES for the combined treatment for all symptoms. Such a tendency would lie in contrast to previous studies, which found NF and MPH was associated with the best improvements in the core symptoms of ADHD [11].

The results of the present study support the use of NF as an alternative treatment for ADHD, especially in the $20 \%$ of children with ADHD who do not respond to medications. In addition, findings from this study support previous suggestions that medications may be reduced when given in combination with other treatments for ADHD [21].

\section{Conclusions}

NF produced a significant improvement in the core symptoms of ADHD, which was equivalent to the effects produced by $\mathrm{MPH}$, based on parental reports. This supports the use of NF as an alternative therapy for children and adolescents with ADHD.

\section{Key messages}

1. NF improves the core symptoms of ADHD based on parental reports.

1. NF and MPH produce equivalent improvements in the core symptoms of ADHD based on parental reports.

\section{Abbreviations \\ ADHD: Attention-deficit/hyperactivity disorder; NF: Neurofeedback; MED: Medication.}

\section{Competing interests}

The authors declare no potential conflicts of interests with regard to the authorship or publication of this article.

\section{Authors' contributions}

NSD and IBE made substantial contributions to the design, analysis, and interpretation of the data, and they were involved in drafting the manuscript and revising it critically. JA contributed to the statistical analyses and data interpretation. DG acquired the funding and contributed to the study design. All authors read and approved the final manuscript.

\section{Acknowledgments}

We thank the staff at the Child and Adolescent Psychiatry Department of the Helse Fonna Hospital of Haugesund for support in completing this study and collecting the data for this article. We thank the Solveig and Johan P. Sommers Foundation and National Competency Center for AD/HD in Norway for financially supporting this project. The project was further supported by the University of Bergen, Center for Child and Adolescent Mental Health.

\section{Author details}

'Department of Child and Adolescent Psychiatry, Helse Fonna Haugesund Hospital, Vinjesgate 10, Haugesund 5501, Norway. ${ }^{2}$ Center for Child and Adolescent Mental Health, University of Bergen, Bergen, Norway. ${ }^{3}$ Center for 
Clinical Research, Haukeland University Hospital, Bergen, Norway. ${ }^{4}$ Department of Research, Helse Fonna Haugesund Hospital, Haugesund, Norway. ${ }^{5}$ Department of Child and Adolescent Psychiatry, Haukeland University Hospital, Haukeland, Norway. ${ }^{6}$ Department of Clinical Medicine, University of Bergen, Bergen, Norway.

Received: 29 November 2011 Accepted: 18 July 2012

Published: 10 August 2012

\section{References}

1. Monastra VJ, Lynn S, Linden M, Lubar JF, Gruzelier J, LaVaque TJ: Electroencephalographic biofeedback in the treatment of attention-deficit/ hyperactivity disorder. Appl Psychophysiol Biofeedback 2005, 30(2):95-114.

2. Barkley RA: Attention-deficit hyperactivity disorder: A handbood for diagnosis and treatment (2nd ed.). New York: Guilford Press; 1998.

3. Safren SA, Sprich S, Mimiaga MJ, Surman C, Knouse L, Groves M, Otto MW: Cognitive behavioral therapy vs relaxation with educational support for medication-treated adults with ADHD and persistent symptoms: a randomized controlled trial. JAMA 2010, 304(8):875-880.

4. Zwi M, Jones $H$, Thorgaard C, York A, Dennis JA: Parent training interventions for Attention Deficit Hyperactivity Disorder (ADHD) in children aged 5 to 18 years. Cochrane Database Syst Rev 2011, 12. CD003018.

5. Langberg JM, Arnold LE, Flowers AM, Epstein JN, Altaye M, Hinshaw SP, Swanson JM, Kotkin R, Simpson S, Molina BS, et al: Parent-reported homework problems in the MTA study: evidence for sustained improvement with behavioral treatment. I Clin Child Adolesc Psychol 2010, 39(2):220-233.

6. Reeves G, Anthony B: Multimodal treatments versus pharmacotherapy alone in children with psychiatric disorders: implications of access, effectiveness, and contextual treatment. Paediatr Drugs 2009, 11(3):165-169.

7. Barbaresi WJ, Katusic SK, Colligan RC, Pankratz VS, Weaver AL, Weber KJ, Mrazek DA, Jacobsen SJ: How common is attention-deficit/hyperactivity disorder? Incidence in a population-based birth cohort in Rochester, Minn. Arch Pediatr Adolesc Med 2002, 156(3):217-224.

8. Barbaresi W, Katusic S, Colligan R, Weaver A, Pankratz V, Mrazek D, Jacobsen S: How common is attention-deficit/hyperactivity disorder? Towards resolution of the controversy: results from a population-based study. Acta Paediatr Suppl 2004, 93(445):55-59.

9. Wagner KD: Management of treatment refractory attention-deficit/ hyperactivity disorder in children and adolescents. Psychopharmacol Bull 2002, 36(1):130-142.

10. Jensen PS, Arnold LE, Swanson JM, Vitiello B, Abikoff HB, Greenhill LL, Hechtman L, Hinshaw SP, Pelham WE, Wells KC, et al: 3-year follow-up of the NIMH MTA study. J Am Acad Child Adolesc Psychiatry 2007, 46(8):989-1002.

11. Monastra VJ, Monastra DM, George S: The effects of stimulant therapy, EEG biofeedback, and parenting style on the primary symptoms of attention-deficit/hyperactivity disorder. Appl Psychophysiol Biofeedback 2002, 27(4):231-249.

12. Monastra VJ: EEG and neurofeedback findingsin ADHD:AN empirical response. The ADHD Report 2004, 12:5-8.

13. Fox DJ, Tharp DF, Fox LC: Neurofeedback: an alternative and efficacious treatment for Attention Deficit Hyperactivity Disorder. Appl Psychophysiol Biofeedback 2005, 30(4):365-373.

14. Arns M, de Ridder S, Strehl U, Breteler M, Coenen A: Efficacy of neurofeedback treatment in ADHD: the effects on inattention, impulsivity and hyperactivity: a meta-analysis. Clin EEG Neurosci 2009, 40(3):180-189

15. Lubar JO, Lubar JF: Electroencephalographic biofeedback of SMR and beta for treatment of attention deficit disorders in a clinical setting. Biofeedback Self Regul 1984, 9(1):1-23.

16. Bakhshayesh AR, Hansch S, Wyschkon A, Rezai MJ, Esser G: Neurofeedback in ADHD: a single-blind randomized controlled trial. Eur Child Adolesc Psychiatry 2011.

17. Rossiter T: The effectiveness of neurofeedback and stimulant drugs in treating AD/HD: part II. Replication. Appl Psychophysiol Biofeedback 2004, 29(4):233-243.
18. Friel PN: EEG biofeedback in the treatment of attention deficit hyperactivity disorder. Altern Med Rev 2007, 12(2):146-151.

19. Othmer SF: Interhemispheric EEG training. J Neurotherapy 2005, 9:87-96.

20. Lubar J: Quantitative Electroencephalographic Analysis (QEEG) Databases for Neurotherapy: Description,Validation, and Application. Haworth Medical Press 2004

21. Lubar JF, Lubar JO: Neurofeedback Assessment and Treatment for Attention Deficit/Hyperactivity Disorders Introduction to Quantitative EEG and Neurofeedback. New York: Academic Press; 1999:103-141.

22. Egner T, Zech TF, Gruzelier JH: The effects of neurofeedback training on the spectral topography of the electroencephalogram. Clin Neurophysiol 2004, 115(11):2452-2460

23. Leins U, Hinterberger T, Kaller S, Schober F, Weber C, Strehl U: Neurofeedback for children with ADHD: a comparison of SCP- and theta/beta-protocols. Prax Kinderpsychol Kinderpsychiatr 2006, 55(5):384-407.

24. Kaiser DA, Othmer SF: Effect of Neurofeedback on Variables of Attention in a Large Multi-Center Trial. Journal of Neurotherapy 2000, 4(1):5-15.

25. Barkley AR: Continuing concerns about EEG biofeedback/neurofeedback. ADHD Report 1993.

26. Loo SK, Barkley RA: Clinical utility of EEG in attention deficit hyperactivity disorder. Appl Neuropsychol 2005, 12(2):64-76

27. Stjernholm O: Neurofeedback as ADDH therapy. Ugeskr Laeger 2010, 172(33):2221-2225.

28. Gevensleben H, Holl B, Albrecht B, Vogel C, Schlamp D, Kratz O, Studer P, Rothenberger $A$, Moll GH, Heinrich H: Is neurofeedback an efficacious treatment for ADHD? A randomised controlled clinical trial. J Child Psychol Psychiatry 2009, 50(7):780-789.

29. Spencer TJ, Biederman J, Wilens TE, Faraone SV: Novel treatments for attention-deficit/hyperactivity disorder in children. J Clin Psychiatry 2002, 63(Suppl 12):16-22.

30. Fuchs T, Birbaumer N, Lutzenberger W, Gruzelier JH, Kaiser J: Neurofeedback treatment for attention-deficit/hyperactivity disorder in children: a comparison with methylphenidate. Appl Psychophysiol Biofeedback 2003, 28(1):1-12.

31. Kropotov JD, Grin-Yatsenko VA, Ponomarev VA, Chutko LS, Yakovenko EA, Nikishena IS: ERPs correlates of EEG relative beta training in ADHD children. Int J Psychophysiol 2005, 55(1):23-34.

32. Linden M, Habib T, Radojevic V: A controlled study of the effects of EEG biofeedback on cognition and behavior of children with attention deficit disorder and learning disabilities. Biofeedback Self Regul 1996, 21(1):35-49

33. Logemann HN, Lansbergen MM, Van Os TW, Bocker KB, Kenemans JL: The effectiveness of EEG-feedback on attention, impulsivity and EEG: a sham feedback controlled study. Neurosci Lett 2010, 479(1):49-53.

34. Duric NS, Elgen I: Norwegian Children and Adolescents with ADHD - A Retrospective Clinical Study: Subtypes and Comorbid Conditions and Aspects of Cognitive Performance and Social Skills. Adolescent Psychiatry 2011, 1:349-354

35. Duric NS, Elgen I: Characteristics of Norwegian children suffering from ADHD symptoms: ADHD and primary health care. Psychiatry Res 2011, 188(3):402-405.

36. Wechsler D, Corporation P: Wechsler intelligence scale for children. New York: Psychological Corporation New York; 1949.

37. Wechsler D: Manual for the Wechsler Intelligence Scale for Children. New York: Psychological Corporation; 1949.

38. Barkley RA: Defiant Children: Second Edition: A Clinician's Manual for Assessment and Parent Training. Guilford Press 1997.

39. Lubar J: Quantitative Electroencephalographic Analysis (QEEG) Databases for Neurotherapy:Description, Validation, and Application. New York: Haworth Medical Press; 2004

40. Technology T; http://www.thoughttechnology.com.

41. Barkley RA: Defiant Children: Second Edition: A Clinician's Manual for Assessment and Parent Training. New York: Guilford Press; 1997.

42. Morris SB, DeShon RP: Combining effect size estimates in meta-analysis with repeated measures and independent-groups designs. Psychol Methods 2002, 7(1):105-125.

43. Kaiser DA, Othmer O: Effect of Neurofeedback on Variables of Attention in a Large Multi-Center Trial. J Neurotherapy: Investig Neuromodulation, Neurofeedback Appl Neuroscience 2000, 4(1):5-15. 
44. Thompson L, Thompson M: Neurofeedback combined with training in metacognitive strategies: effectiveness in students with ADD. Appl Psychophysiol Biofeedback 1998, 23(4):243-263.

doi:10.1186/1471-244X-12-107

Cite this article as: Duric et al.: Neurofeedback for the treatment of children and adolescents with ADHD: a randomized and controlled clinical trial using parental reports. BMC Psychiatry 2012 12:107.

\section{Submit your next manuscript to BioMed Central} and take full advantage of:

- Convenient online submission

- Thorough peer review

- No space constraints or color figure charges

- Immediate publication on acceptance

- Inclusion in PubMed, CAS, Scopus and Google Scholar

- Research which is freely available for redistribution 